\title{
Optimizing the data available via Health Canada's clinical information portal
}

\author{
Alexander C. Egilman BA, Joseph S. Ross MD MHS, Matthew Herder LLM JSM
}

Cite as: CMAJ 2021 August 23;193:E1305-6. doi: 10.1503/cmaj.210877

$\mathbf{T}$ hrough its Public Release of Clinical Information (PRCI) initiative, Health Canada has provided public access to a vast repository of data that have been submitted to support market authorization of drugs and medical devices. ${ }^{1}$ These are published on the agency's online portal (https://clinical -information.canada.ca/search/ci-rc), which is free to access, easy to use and contains a rapidly expanding list of medical products for which data are available. ${ }^{2}$ It is unlikely that most patients and clinicians will make use of the clinical data and metadata available through Health Canada's portal; therefore, their greatest potential value is in secondary research on regulatory decision-making, development of clinical practice guidelines and assessment of health technologies. Yet, whether the research community, physicians and others will take full advantage of these data remains to be seen.

Since the PRCI launched in March 2019, Health Canada has released data from more than 160 submissions for drugs, biologics, vaccines and medical devices. The regulator is currently in its third year of a 4-year phase-in schedule to release clinical data proactively from submissions for all new active substances, new clinical indications, generic drugs and higher-risk devices that are approved, withdrawn or rejected. Substantial clinical data submitted by the industry sponsor of the application, including summary-level data (e.g., clinical overviews, clinical summaries and clinical study reports) and metadata (e.g., trial protocols and statistical analysis plans) are made publicly available by Health Canada as a matter of policy.

Health Canada is currently an outlier among medical regulators in releasing these clinical reports proactively. ${ }^{3}$ The European Medicines Agency (EMA) suspended proactive publication of clinical reports in 2018, citing cuts in staff caused by Britain's exit from the European Union (Brexit) and its relocation to Amsterdam. The US Food and Drug Administration (FDA) ended its Clinical Data Summary Pilot Program in 2020 after only 1 company volunteered to participate. Notably, the EMA and FDA continue to publish "action packages" proactively, which contain medical and statistical reviews and other regulator-generated documents, but do not include information prepared by drug sponsors, most critical of which are the clinical study reports.

The same sponsor-submitted clinical data for older drug and medical device applications (i.e., approved, withdrawn or rejected

\section{Key points}

- Since Health Canada launched its Public Release of Clinical Information (PRCI) initiative in 2019, the agency has released clinical data from more than 160 submissions for drugs, biologics, vaccines and medical devices.

- Extensive data, including clinical study reports that support each submission for a medical product, are now freely accessible on the agency's online portal, and older drug data are available upon request.

- Use of the data appears low; increased use of these data could be invaluable, including in the development of clinical practice guidelines and analyses of cost effectiveness of drugs and devices, and to advance knowledge about the risks and benefits of medical products.

before March 2019) are also available upon request through the Health Canada online portal. Requesting these data is straightforward: anyone, regardless of geographic location or nationality, can request and access data at no cost. Through April 2021, Health Canada has published data from 70 submissions for drugs, biologics, vaccines and medical devices in response to information requests, which took 4.5 months to process on average, much faster than comparable requests to the EMA and the FDA. ${ }^{3}$ Given that sponsors typically submit similar data to regulators, Health Canada's portal may be the most efficient source of clinical data submitted to the FDA, EMA and elsewhere.

Investigators and research groups involved in evidence assessment, such as Cochrane Review Groups and EvidenceBased Practice Centers, may use the data to reproduce and validate original analyses, carry out secondary analyses to explore new research questions and conduct systematic reviews and meta-analyses by synthesizing results from multiple trials. ${ }^{4}$ For example, clinical study reports provide substantially more detailed information on the design, conduct and results of clinical trials than publications, facilitating identification of errors and gaps in the published literature, and making them the preferred information source for systematic reviews and metaanalyses. ${ }^{5}$ Previous critical reviews and meta-analyses using clinical study reports have found that widely used interventions, such as oseltamivir (an antiviral) and reboxetine (an antidepressant), 
were ineffective or unsafe..$^{5-7}$ However, most systematic reviewers do not use these reports, with many citing time and resource constraints as key barriers to access and use. ${ }^{8}$

The development of rigorous, trustworthy clinical practice guidelines depends on high-quality systematic reviews of evidence. Including unpublished trial data in systematic reviews is critical to minimizing reporting and publication biases, and may even change conclusions about the benefits and risks of treatments. ${ }^{6,7}$ However, fewer than half of systematic reviews search for unpublished data, despite guidance to include unpublished data from the Institute of Medicine. ${ }^{9}$ Health Canada's PRCI initiative could serve as an important resource for identifying and integrating unpublished data into systematic reviews for those developing clinical practice guidelines.

Review of data on Health Canada's portal may also help inform cost-effectiveness analyses. Clinical study reports are key sources of information for several health technology assessment agencies (e.g., Canadian Agency for Drugs and Technologies in Health); however, other agencies (e.g., the Institute for Clinical and Economic Review) may benefit from greater use of clinical study reports in value assessments.

Although Health Canada provides clinical reports on submissions for more than 160 medical products, the initiative has limitations. Patient-level data are excluded from proactive disclosure for privacy reasons, making it impossible to carry out a complete reanalysis of a clinical trial. In addition, clinical information not used by the sponsor to support the proposed conditions of use are exempt from disclosure. However, Health Canada determines whether redactions proposed by manufacturers are justified, and a recent analysis found that most redactions were minor. ${ }^{3}$

The primary factor limiting the impact of the $\mathrm{PRCl}$ initiative appears to be underuse. We are aware of only 1 published study that used data from the Health Canada portal. ${ }^{10}$ Increasing awareness and use of Health Canada's portal through increased funding for secondary research (e.g., grants dedicated for research using shared data sources), and greater appreciation of replication studies and secondary analyses among academic promotion and journal editorial committees, may be necessary.

Health Canada's PRCl initiative has potential to advance scientific knowledge and strengthen the evidence base for regulatory and clinical decisions. However, until awareness and use increase, its full benefits will not be realized.

\section{References}

1. Guidance document on Public Release of Clinical Information: profile page. Ottawa: Health Canada; modified 2019 Mar. 29. Available: https://www.canada.ca /en/health-canada/services/drug-health-product-review-approval/profile-public -release-clinical-information-guidance.html (accessed 2021 July 2).

2. Search for clinical information on drugs and medical devices. Ottawa: Health Canada; modified 2019 May 29. Available: https://clinical-information.canada. ca/search/ci-rc (accessed 2021 July 2).

3. Egilman AC, Kapczynski A, McCarthy ME, et al. Transparency of regulatory data across the European Medicines Agency, Health Canada, and US Food and Drug Administration. J Law Med Ethics. In press.

4. National Academies of Sciences, Engineering, and Medicine. Reflections on sharing clinical trial data: challenges and a way forward - Proceedings of a workshop. Washington (DC): National Academies Press; 2020.

5. Doshi P, Jefferson T, Del Mar D. The imperative to share clinical study reports: recommendations from the Tamiflu experience. PLoS Med 2012;9:e1001201.

6. Eyding D, Lelgemann M, Grouven U, et al. Reboxetine for acute treatment of major depression: systematic review and meta-analysis of published and unpublished placebo and selective serotonin reuptake inhibitor controlled trials. BMJ 2010;341:c4737.

7. Chan A-W, Song F, Vickers A, et al. Increasing value and reducing waste: addressing inaccessible research. Lancet 2014;383:257-66.

8. Hodkinson A, Dietz KC, Lefebvre C, et al. The use of clinical study reports to enhance the quality of systematic reviews: a survey of systematic review authors. Syst Rev 2018;7:117.

9. Institute of Medicine. Finding what works in health care: standards for systematic reviews. Washington (DC): The National Academies Press; 2011.

10. Branson J, Good N, Chen J-W, et al. Evaluating the re-identification risk of a clinical study report anonymized under EMA Policy 0070 and Health Canada Regulations. Trials 2020;21:200.
Competing interests: Matthew Herder is a member of the Patented Medicine Prices Review Board (Canada's national drug price regulator) and has received honoraria for his public service. No other competing interests were declared.

This article has been peer reviewed.

Affiliations: Center for Outcomes Research and Evaluation (Egilman, Ross), Yale-New Haven Hospital; Department of Internal Medicine (Ross), Yale School of Medicine; Department of Health Policy and Management (Ross), Yale School of Public Health, New Haven, Conn.; Health Law Institute (Herder), Schulich School of Law, and Department of Pharmacology (Herder), Faculty of Medicine, Dalhousie University, Halifax, NS.

Contributors: All of the authors contributed to the conception and design of the work, drafted the manuscript, revised it critically for important intellectual content, gave final approval of the version to be published and agreed to be accountable for all aspects of the work.

Funding: Matthew Herder has received grants from the Canadian Institutes of Health Research (PJT 156256 and CMS 171741), paid to his institution. Alexander Egilman has received research support from the Laura and John Arnold Foundation at Yale University (Collaboration for Research Integrity and Transparency). Alexander Egilman and Joseph Ross have received research support from the US Food and Drug Administration for the YaleMayo Clinic Center for Excellence in Regulatory Science and Innovation program (U01FD005938). Joseph Ross has received research support from Johnson \& Johnson to develop methods of clinical trial data sharing; from the Medical Device Innovation Consortium as part of the National Evaluation System for Health Technology, from the Agency for Healthcare Research and Quality (R01HS022882); from the National Heart, Lung and Blood Institute of the National Institutes of Health (R01HS025164, R01HL144644); and from the Laura and John Arnold Foundation to establish both the Good Pharma Scorecard at Bioethics International and the Collaboration for Research Integrity and Transparency at Yale.

Content licence: This is an Open Access article distributed in accordance with the terms of the Creative Commons Attribution (CC BY-NC-ND 4.0) licence, which permits use, distribution and reproduction in any medium, provided that the original publication is properly cited, the use is noncommercial (i.e., research or educational use), and no modifications or adaptations are made. See: https://creativecommons.org/licenses/ by-nc-nd/4.0/

Correspondence to: Matthew Herder, matthew.herder@dal.ca 